\title{
Efeito do feedback visual na força isométrica da pinça de três dedos
}

\section{Effect of visual feedback in the isometric force of the pinch of three fingers}

\author{
Ronaldo Luis da Silval; Renata Escorcio²; Michele Emy Hukuda ; Lilian Aparecida Yoshimura \\ Fernandes2; Eduardo Vital de Carvalho3; Fátima Aparecida Caromano ${ }^{4}$ \\ ${ }^{1}$ Mestre em Ciências da Reabilitação - USP. Professor do curso de fisioterapia - Uniban. \\ ${ }^{2}$ Mestre em Ciências da Reabilitação - USP. \\ ${ }^{3}$ Fisioterapeuta da Associação Brasileira de Distrofia Muscular. Especialista em intervenção fisioterapêutica nas doenças \\ neuromusculares - Unifesp/EPM \\ Professora Doutora do curso de Fisioterapia da Faculdade de Medicina - USP.
}

\section{Endereço para correspondência}

Curso de Fisioterapia da FM- USP

Laboratório de Fisioterapia e Comportamento - LaFi.Com

R. Cipotânea, 51 - Cidade Universitária - Butantã

05360-000 - São Paulo - SP [Brasil]

fcaromano@uol.com.br

\begin{abstract}
Resumo
A função e a força muscular são dois parâmetros frequentemente avaliados durante o exame neurológico pediátrico. A avaliação da força isométrica, por meio da dinamometria manual, é uma técnica de fácil aplicação. Nesta pesquisa, objetiva-se avaliar a influência do feedback visual nos dados gerados pela dinamometria manual em crianças saudáveis, para aperfeiçoar a metodologia do teste. Mensurou-se a força máxima isométrica por meio de um dinamômetro manual na atividade de pinça de três dedos sem e com feedback visual. Não foram encontradas diferenças estatisticamente significantes entre os dados obtidos sem e com feedback visual, bem como influência da idade, do peso, da altura e do IMC. Sugerem-se mais estudos com população similar e com adultos e crianças portadores de necessidades especiais. Concluiu-se que o feedback visual não afeta os dados obtidos nos teste de força isométrica de pinça de três dedos.
\end{abstract}

Descritores: Criança; Dinamômetro; Força muscular; Força de pinça;

Retroalimentação psicológica.

\begin{abstract}
The function and the muscular strength are two parameters frequently appraised during the pediatric neurological exam. The isometric method, through the manual dynamometry, is a technique of easy application. The objective of this study is to evaluate the influence of the visual feedback in the data generated in the test of isometric maximum force by the manual dynamometry in a sample of healthy children. The isometric maximum force was measured through a manual dynamometer in the activity of pinch of three fingers with and without visual feedback. Differences statistically significant were not observed among the data obtained without and with visual feedback, as well as influence of age, weight, height, and IMC. More studies are suggested with similar population and with adults and disabled children. It was concluded that the visual feedback do not influence the data generated in the test of isometric maximum force by the manual dynamometry of pinch of three fingers.
\end{abstract}

Key words: Child; Dynamometer; Feedback; Muscle strength; Pinch strength. 


\section{Introdução}

Função e força muscular são dois parâmetros frequentemente avaliados durante o exame neurológico pediátrico, sendo essencial para avaliação do sistema motor ${ }^{1,2}$.

Valores de referência para força isométrica de pinça de três dedos auxiliam na determinação da evolução de doenças neurológicas e musculares, especialmente as progressivas ${ }^{2,3,4}$.

Dois métodos de avaliação de força se sobressaem na pesquisa clínica, o exame isocinético e o isométrico. O primeiro é dependente de equipamento sofisticado e o segundo é normalmente realizado por meio da dinamometria manual, técnica de fácil aplicação, baixo custo, além de ser rotina de exame estabelecida e validada ${ }^{5,6,7,8}$.

Muitos fatores interferem no controle da força manual em crianças, principalmente maturação estrutural do sistema neuromotor e sensitivo, peso, altura, idade, nutrição, herança genética, prática de atividade física e integridade muscular ${ }^{3}$.

Existe registro de que o feedback visual afeta os resultados de testes isocinéticos concêntricos, tanto em baixa velocidade quanto em alta velocidade angular ${ }^{9}$, embora outro estudo tenha demonstrado que tal influência foi reduzida após três repetições ${ }^{10}$. Entretanto, não se encontraram trabalhos que analisassem a influência do feedba$c k$ visual na realização de testes de força muscular isométrica realizados por meio da dinamometria manual. Em decorrência da ampla utilização da dinamometria manual, a investigação da influência do feedback visual ganha uma relevância significativa no refinamento do método de coleta de dados. Neste estudo, objetivou-se avaliar a influência do feedback visual nos dados gerados no teste de força máxima isométrica pela dinamometria manual em uma amostra de crianças e adolescentes saudáveis.

\section{Materiais e métodos}

\section{Participantes}

Os 46 participantes que compuseram a amostra eram alunos do sexo masculino, destros, entre 8 e 15 anos, regularmente matriculados em uma escola particular de ensino fundamental da cidade de São Paulo. Foram fatores de exclusão o histórico de doenças musculoesqueléticas, ortopédicas, neuromusculares ou deficiência visual. Todos os participantes, em conjunto com seus responsáveis, assinaram o Termo de Consentimento Livre e Esclarecido, conforme aprovado pelo Conselho de Ética. Este estudo é parte do projeto intitulado "Elaboração, validação e aplicação de escala de avaliação funcional para portadores de distrofia muscular de Duchenne (DMD)", aprovado pela Comissão de Ética para Análise de Projetos de Pesquisa do Hospital das Clínicas da Faculdade de Medicina da Universidade de São Paulo, número 837/05, e foi executado com a finalidade de refinar o método de avaliação da força máxima isométrica.

A dominância em membros superiores foi determinada por meio de cinco questões, as quais deveriam ser respondidas de forma verbal e gestualmente, mimetizando a realização da tarefa requisitada. O participante era questionado a respeito de qual era sua mão principal e qual utilizava para escrever, tomar sopa, escovar dentes e sacar uma bola. Era necessário que ao menos três respostas coincidissem para determinar a mão dominante.

\section{Procedimentos}

Mensurou-se a força máxima isométrica por meio de um dinamômetro manual (Nicholas MMT, Laffayette Inc ${ }^{\circledR}$, USA) da atividade de pinça de três dedos (polegar contra indicador e médio). Os participantes eram posicionados em sedestação, com pés apoiados no chão e cotovelos sobre a mesa de avaliação. Os testes foram realizados pelo mesmo examinador, ajudado por fisioterapeuta auxiliar no intuito de prevenir compensações posturais por parte dos participantes durante as provas. Realizaram-se duas medições em cada mão por meio do método make-test ${ }^{11}$, com intervalo de 15 segundos entre elas, sem que fosse permitido ao participante observar o visor do dinamômetro durante a execução dos testes. 
Após 3 minutos, repetiu-se o teste mais duas vezes, permitindo-se, dessa vez, que o participante acompanhasse o resultado no visor ${ }^{12}$. Em ambas as situações de teste, a primeira medição prestouse ao treinamento do exercício, e a segunda, para coleta dos dados ${ }^{11,13}$.

\section{Ānólise de dados}

Foi utilizada estatística descritiva e, para comparação entre dois grupos de medidas, utilizou-se o teste " $\mathrm{t}$ " Student pareado. Inicialmente, os dados foram analisados em razão da dominância manual dos participantes, comparando-se os resultados obtidos sem e com feedback visual. Posteriormente, para avaliar a influência da idade, do peso, da altura e do índice de massa corpórea (IMC) no experimento, dividiu-se o grupo dos participantes destros em quartis.

\section{Resultados}

Os dados referentes à caracterização da amostra são apresentados na Tabela 1. Não foram encontradas diferenças estatisticamente significantes entre os resultados obtidos sem e com feedback visual, tanto para o membro superior direito quanto esquerdo ( $p=0,516$ e 0,948$)$. Realizou-se a divisão do subgrupo de participantes destros em quartis para análise da influência da idade, do peso, da altura e do IMC nos dados obtidos.

Essas análises também não demonstraram diferenças estatisticamente significantes entre as medidas realizadas sem e com feedback visual para as variáveis pesquisadas (Tabela 2).

Tabela 1: Características dos participantes do estudo

\begin{tabular}{c|c|c|c|c}
\hline & Idade & Peso (kg) & Altura (m) & IMC \\
\hline Mínimo & $8^{\mathrm{a}}$ & 24,70 & 124,0 & 15,8 \\
\hline Máximo & $12 \mathrm{a} 2 \mathrm{~m}$ & 83,00 & 173,0 & 29,4 \\
\hline Média & $10 \mathrm{a} 3 \mathrm{~m}$ & 46,50 & 152,3 & 19,6 \\
\hline DP & $1 \mathrm{a} 2 \mathrm{~m}$ & 13,54 & 14,6 & 3,0 \\
\hline
\end{tabular}

\section{Discussão}

O valor de uma técnica de avaliação depende de sua fidelidade em descrever uma determinada variável. Assim, investigar e qualificar os possíveis fatores de influência nos resultados obtidos, as variáveis que afetam o dado, por meio dessa técnica é de fundamental importância. Neste estudo, verificou-se que não houve influência do feedback visual nos resultados obtidos para mensuração da força máxima isométrica pela dinamometria manual nos participantes. Entretanto, não foi possível obter uma resposta mais concreta quanto aos participantes sinistros em razão do número reduzido na amostra, e por terem sido investigados indivíduos normais nesta pesquisa.

Estudos que analisem a influência do $f e-$ edback visual em indivíduos com deficiência de força muscular de etiologia miopática ou neuropática carecem ainda de investigação. Kellis e Baltzopoulos ${ }^{9}$ fazem uma revisão de estudos que demonstram essa influência nos resultados dos testes isocinéticos, conduzidos em diferentes velocidades angulares. Kim e Kramer ${ }^{10}$, entretanto, ao observarem que essa influência nos resultados obtidos no teste isocinético diminuiu após certo número de repetições, sugeriram que tal achado se relacionasse à aprendizagem motora. Embora se tratem de testes diferentes, tal explicação pode estar relacionada aos resultados encontrados neste estudo. Dado que as populações de pesquisa dos artigos relacionados e deste trabalho não são similares, sugere-se estudo avaliando a influência do feedback visual nos valores do teste de força máxima isométrica por meio da dinamometria manual em indivíduos adultos.

\section{Conclusão}

Este estudo demonstrou que o feedback visual, propiciado por meio do acesso aos dados mostrados no visor do dinamômetro, durante a realização do teste de força máxima isométrica, em crianças entre 8 e 12 anos, não apresenta influência estatisticamente significativa, indepen- 
Tabela 2: Diferenças estatísticas ${ }^{\star}$ entre medidas obtidas sem e com feedback dos membros direito e esquerdo nas diferentes faixas etórias

\begin{tabular}{|c|c|c|c|c|c|c|c|c|}
\hline & \multicolumn{2}{|c|}{$1^{\circ}$ quartil } & \multicolumn{2}{|c|}{$2^{\circ}$ quartil } & \multicolumn{2}{|c|}{$3^{\circ}$ quartil } & \multicolumn{2}{|c|}{$4^{\circ}$ quartil } \\
\hline & D & $E$ & D & $\mathbf{E}$ & D & $\mathbf{E}$ & D & $E$ \\
\hline \multirow{2}{*}{ Idade } & \multicolumn{2}{|c|}{$8-11$ anos } & \multicolumn{2}{|c|}{$11-12$ anos } & \multicolumn{2}{|c|}{$12-14$ anos } & \multicolumn{2}{|c|}{$14-15$ anos } \\
\hline & $p=0,191$ & $p=0,620$ & $p=0,094$ & $p=0,966$ & $p=0,352$ & $p=0,651$ & $p=0,913$ & $p=0,512$ \\
\hline \multirow{2}{*}{ Peso } & \multicolumn{2}{|c|}{$24-35 \mathrm{~kg}$} & \multicolumn{2}{|c|}{$35-47 \mathrm{~kg}$} & \multicolumn{2}{|c|}{$47-54,4 \mathrm{~kg}$} & \multicolumn{2}{|c|}{$54,5-83 \mathrm{~kg}$} \\
\hline & 0,828 & 0,886 & 0,393 & 0,386 & 0,846 & 0,250 & 0,260 & 0,715 \\
\hline \multirow{2}{*}{ Altura } & \multicolumn{2}{|c|}{$1,24-1,41 \mathrm{~m}$} & \multicolumn{2}{|c|}{$1,41-1,55 m$} & \multicolumn{2}{|c|}{$1,55-1,65 \mathrm{~m}$} & \multicolumn{2}{|c|}{$1,65-1,73 m$} \\
\hline & 0,533 & 0,685 & 0,499 & 0,166 & 0,061 & 0,336 & 0,944 & 0,974 \\
\hline \multirow{2}{*}{ IMC } & \multicolumn{2}{|c|}{$15,8-17,3$} & \multicolumn{2}{|c|}{$17,3-18,8$} & \multicolumn{2}{|c|}{$18,8-22$} & \multicolumn{2}{|c|}{$22-29,4$} \\
\hline & 0,675 & 0,965 & 0,268 & 0,840 & 0,120 & 0,279 & 0,484 & 0,461 \\
\hline
\end{tabular}

* Diferença estudada por meio do teste " $\mathrm{t}$ " Student, considerando-se significativos valores iguais ou inferiores a 0,05

dentemente da idade, do peso, da altura e do IMC do indivíduo avaliado.

\section{Referências}

1. Van der Ploeg RJO, Fidler V, Oosterhuis HJGH. Hand-held dynmometry: reference values. J Neurol Neurosurg Psychiatry. 1991;54:244-7.

2. Beenaker EAC, Van Der Hoeven JH, Fock JM, Maurits NM. Reference values of maximum isometric muscle force obtained in 270 children aged 4-16 years by hand-held dynamometry. Neuromuscul Disord. 2001;11:441-6.

3. Smits-Engelsman BCM, Westenberg Y, Duysens J. Development of isometric force and force control in children. Brain Res Cogn Brain Res. 2003;17(1):68-74.

4. Van Den Beld WA, Van Der Sander GAC, Sengers RCA, Verbeek ALM, Grabeels FJM. Validity and Reproducibility of hand-held dynamometry and children aged 4-11 years. J Rehabil Med. 2006;38:57-64.

5. Hosking JP, Bhat US, Dubowitz V, Edwards RH. Measurements of muscle strength and performance in children with normal and diseased muscle. Arch Dis Child. 1976;51:957-63.

6. Backman,E, Odenrick P, Henriksson KG, Ledin T. Isometric muscle force and anthropometric values in normal children aged between 3.5 and 15 years. Scand J Rehabil Med. 1989;21:105-14.
7. Backman E, Johansson V, Hager B, Sjoblom P, Henriksson KG. Isometric muscle strength and muscular endurance in normal persons aged between 17 and 70 years. Scand J Rehab Med. 1995;27:109-17.

8. Zeevi D. The measurement of isokinetic fingers flexion strength. Clin Biomech. 1998;12:473-81.

9. Kellis E, Baltzopoulos V. Resistive eccentric exercise: effects of visual feedback on maximum moment of knee extensors and flexors. J Orthop Sports Phys Ther. 1996; 23(2):120-4.

10. Kim HJ, Kramer JF. Effectiveness of visual feedback during isokinetic exercise. J Orthop Sports Phys Ther. 1997;26(6):318-23.

11. Caromano FA, Durigon OFS, Ide MR, Pardo MS, Candeloro JM, Yamagata RA, et al. Make-test and break-test in isometric muscle strength measurement of knee extensor muscles in elder women. Revista Salusvita, Bauru - SP. 2004;23(3):423-7.

12. Wolf SL, Binder-Macleod SA. Neurophysiological factors in electromyographic feedback for neuromotor disturbances. In: Basmajian JV, Editor. Biofeedback: principles and practice for clinicians. $3^{\text {a }}$ ed. Baltimore: Williams \& Wilkins, 1989.

13. Bohannon RW. Reference values for extremity muscle strength obtained by hand-held dynamometry from adults aged 20 to 79 years. Arch Phys Med Rehabil. 1997;78:26-32. 